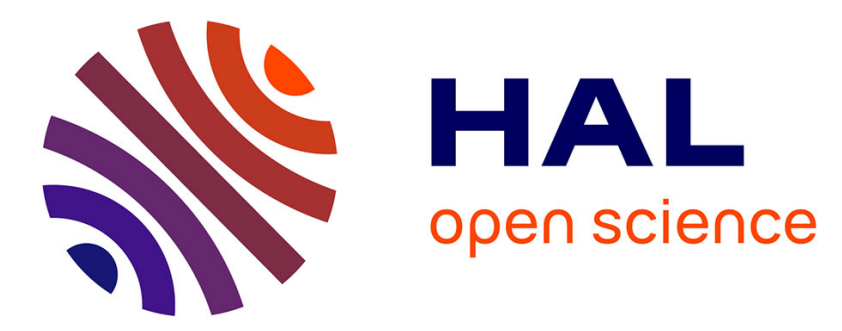

\title{
Diffraction stress analysis of grain interaction in polycrystalline materials
}

\author{
U. Welzel, Sylvain Fréour, A. Kumar, E. J. Mittemeijer
}

\section{To cite this version:}

U. Welzel, Sylvain Fréour, A. Kumar, E. J. Mittemeijer. Diffraction stress analysis of grain interaction in polycrystalline materials. Zeitschrift für Kristallographie, 2006, S23, pp.43-49. 10.1524/zksu.2006.suppl_23.43 . hal-01006745

\section{HAL Id: hal-01006745 \\ https://hal.science/hal-01006745}

Submitted on 20 Jan 2018

HAL is a multi-disciplinary open access archive for the deposit and dissemination of scientific research documents, whether they are published or not. The documents may come from teaching and research institutions in France or abroad, or from public or private research centers.
L'archive ouverte pluridisciplinaire $\mathbf{H A L}$, est destinée au dépôt et à la diffusion de documents scientifiques de niveau recherche, publiés ou non, émanant des établissements d'enseignement et de recherche français ou étrangers, des laboratoires publics ou privés. 


\title{
Diffraction stress analysis of grain interaction in polycrystalline materials
}

\author{
U. Welzel*, S. Fréour, A. Kumar, E.J. Mittemeijer \\ Max Planck Institute for Metals Research, Heisenbergstr. 3, 70569 Stuttgart, Germany \\ * Contact author; e-mail: u.welzel@mf.mpg.de
}

Keywords: grain interaction, stress analysis, surface anisotropy, grain-shape texture

\begin{abstract}
This work summarises recent developments of so-called direction-dependent elastic grain-interaction models. The notion 'direction-dependent' grain-interaction signifies that different grain-interaction constraints prevail along different directions in a specimen. Practical examples of direction-dependent grain interaction are the occurrence of surface anisotropy in thin films (and, possibly, surface regions of bulk polycrystals) and the occurrence of a grain-shape (morphological) texture.
\end{abstract}

\section{Introduction}

Elastic grain-interaction models describe the distribution of stresses and strains over the (crystallographically) differently oriented grains of a mechanically stressed polycrystal and allow the calculation of mechanical and diffraction (X-ray) elastic constants (diffraction (Xray) stress factors for mechanically elastically anisotropic samples, relating (diffraction) lattice strains to mechanical stresses [1,2]), from single-crystal elastic data. Usually, the grain-interaction models proposed by Voigt, Reuss, Neerfeld-Hill or Eshelby-Kröner are employed [1]. These models devised for bulk polycrystals with an isotropic microstructure imply that (in the absence of crystallographic texture) a polycrystal as a whole is macroscopically elastically isotropic. They have therefore been termed isotropic graininteraction models' [2]. However, polycrystals cannot generally be considered as being macroscopically elastically isotropic. Two cases of direction-dependent (anisotropic) grain interaction can be distinguished: the case of surface anisotropy and the case of a grain-shape texture.

Surface anisotropy: In a (columnar) thin film (or the surface layer of a bulk polycrystal), each crystallite is surrounded by neighbouring crystallites in only two dimensions. Thus, the grain-interaction perpendicular to the surface can be different from the grain interaction parallel to the surface: direction-dependent (anisotropic) grain interaction occurs [3-7].

Grain-shape texture: It appears obvious that deviations from an isotropic 'microstructure', as for example due to the reduced dimensionality of a thin film, may generally have an impact on the grain interaction. Following this line of reasoning, it can be anticipated that a polycrystal composed of non-spherical grains with their principal axes being aligned more or less preferentially along certain directions in the specimen (i.e., a grain-shape or 
morphological texture occurs) exhibits macroscopically elastically anisotropic behaviour too, i.e. also in this case anisotropic grain-interaction occurs [9]. This work summarises recent developments of direction-dependent elastic grain-interaction models, presents a comparison and an experimental example.

\section{Theoretical background}

\section{Thin films / surface anisotropy [3-7]}

In the following, an at least transversely elastically isotropic polycrystal is considered. Graininteraction assumptions taking into account that in (columnar) thin films and surface layers of bulk polycrystals, neighbouring grains surround a grain in only two dimensions can, for example, be formulated as follows (cf. also [8]): (i) in the plane of the film, the strains are equal for all crystallites and (ii) the stresses perpendicular to the layer are identical for all crystallites. These grain-interaction assumptions fix certain stress and strain tensor components to corresponding mechanical average values (mechanical averages are indicated by brackets $<>$ ):

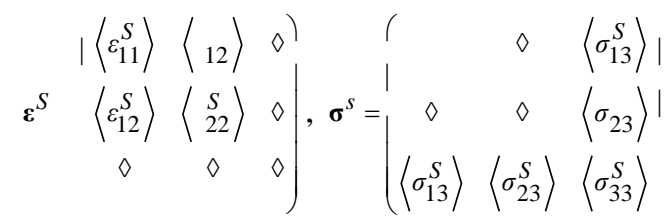

The superscript $S$ indicates that a tensor is expressed in the specimen frame of reference $S$. The tensor components marked by $\diamond$ are not explicitly specified for every crystallite, but these components can be calculated from Hooke's law for every crystallite, $\varepsilon_{i j}^{S}=s_{i j k l}^{S} \sigma_{k l}^{S}$. The $s_{i j k l}^{S}$ are the single crystal elastic compliances. Hooke's law represents a system of nine equations for eighteen unknowns, but as the strain $\varepsilon$ and stress $\boldsymbol{\sigma}$ tensors are symmetric (i.e. $\varepsilon_{i j}=\varepsilon_{j i}$ and $\sigma_{i j}=\sigma_{j i}$ ), Hooke's law is a short notation for six independent equations for twelve independent unknowns. If six components of the twelve unknowns are known, as a consequence, the other components can be calculated by solving Hooke's law.

Extreme grain-interaction assumptions (e.g. the stresses perpendicular to the layer are identical for all crystallites) are adopted in the above described Vook-Witt (VW) model. In terms of the bulk models it could be said that, Voigt behaviour ('equality of strains') is attributed to the in-plane directions, whereas Reuss behaviour ('equality of stresses') is attributed to the direction perpendicular to the film surface. Thus, the Vook-Witt model will generally be incompatible with the true elastic behaviour of a polycrystal. To overcome this problem, the inverse Vook-Witt model (iVW), required for the construction of an effective grain-interaction model, has been proposed $[2,5,6]$. The grain-interaction assumptions for the inverse Vook-Witt model are as follows: (i) the in-plane stress is equal for all crystallites and (ii) the strain perpendicular to the film surface is equal for all crystallites: 


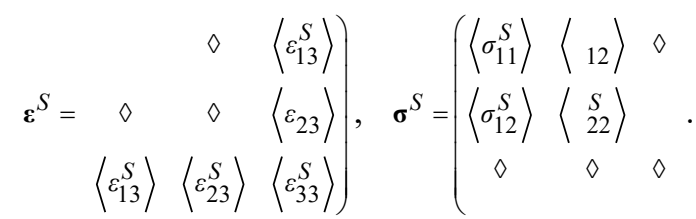

The tensor components marked by $\diamond$ are not explicitly specified for every crystallite, but these components can be calculated, as described above for the Vook-Witt model.

The above formulated grain-interaction assumptions allow the calculation of the mechanical elastic constants and the diffraction stress factors.

The need for introducing the inverse Vook-Witt model can be understood as follows: The number of grain-interaction models of extreme types of grain-interaction assumptions is two for bulk materials (Reuss and Voigt). However, four types of extreme grain-interaction models then can be formulated for (columnar) thin films, as two principal directions, each with two extreme grain-interactions, occur. These extreme grain-interaction models are the Reuss, the Voigt, the Vook-Witt and the inverse Vook-Witt models. In a so-called effective grain-interaction model, the mechanical and diffraction elastic constants (or diffraction stress factors) are calculated as arithmetic weighted averages of the elastic constants obtained from a set of extreme grain-interaction models. A well known example for an effective graininteraction model is the Neerfeld-Hill model (= 'average' of 'Reuss' and 'Voigt', in case of isotropic grain interaction). Here, a weighted average of the Reuss, Voigt, Vook-Witt and inverse Vook-Witt models is proposed for cases of anisotropic grain interaction. The background of any averaging of (extreme) grain-interaction models to describe physical reality could be described as follows: A real sample is conceived to be constituted from separate volume fractions of crystallites, each of which obeys a certain type of graininteraction.

\section{Grain-shape (morphological) texture [9]}

In order to calculate the elastic constants of a polycrystal from single-crystal elastic data in the Eshelby-Kröner (EK) model, the crystallites surrounding an individual grain (inclusion) in a polycrystal are conceived as an elastically homogenous matrix with the elastic properties of the entire polycrystal. Traditionally, a spherical shape of the inclusions is considered [1]. A grain-shape (morphological) texture can be incorporated in the Eshelby-Kröner model by considering ellipsoidal inclusions with their principal axes aligned along common directions in the specimen frame of reference. The shape of the crystallites can be described by a shape parameter $\eta$, which is defined as the ratio of the principal axis of the ellipsoid in the zdirection $\left(a_{3}\right)$ of the specimen frame of reference and the principal axes of the ellipsoid in the x-direction $\left(a_{1}\right)$ and the y-direction $\left(a_{2}\right)$ in the specimen frame of reference, respectively: $\eta=a_{3} / a_{1}=a_{3} a_{2}$. Thus, the considered ellipsoids exhibit rotational symmetry with respect to the surface normal of the specimen.

Diffraction stress analysis and diffraction analysis of elastic grain interaction The unknown stress tensor components $\left\langle\sigma_{i j}^{s}\right\rangle$ can be obtained as fit parameters in a (leastsquares) minimisation of the difference $\chi^{2}$, 


$$
\chi^{2}=\sum_{i} \omega_{i}^{2}\left(\varepsilon_{i}^{\text {calc }}\left(\left\langle\sigma^{s}\right\rangle, h k l, \varphi, \psi\right)-\varepsilon_{i}^{\text {meas }}(h k l, \varphi, \psi)\right)^{2}
$$

where the $\varepsilon_{i}^{\text {meas }}$ represent the measured lattice strains. The index $i$ stands for all lattice strains determined from measurements (for all different $\psi, \varphi$ and/or $h k l$ analysed). The $\omega_{i}$ are weighing factors which can be used to reflect the statistical relevance of the measured strains $\varepsilon_{i}^{\text {meas }}(h k l, \varphi, \psi)$ in the result of the minimisation and could correspond to the inverse of the standard deviations of the measured strains. For the case of macroscopically elastically anisotropic specimens considered here, the diffraction strains, $\varepsilon_{i}^{\text {calc }}$, have to be calculated from

$$
\varepsilon_{i}^{\text {calc }}\left(\left\langle\sigma^{s}\right\rangle, h k l, \varphi, \psi\right)=F_{m n}(\psi, \varphi, h k l)\left\langle\sigma_{m n}^{s}\right\rangle,
$$

where the $F_{m n}(\psi, \varphi, h k l)$ are the so-called diffraction (X-ray) stress factors [1,2]. The following variant of the above-described method has been proposed [5,6]: In addition to the unknown stress tensor components, parameters related to the grain interaction can be refined in the fitting procedure. If the elastic grain interaction in the specimen can be parameterized by parameters $f_{\mathrm{i}}$, which are not known a priori, they can be used as fitting parameters in addition to the unknown stress tensor components. Such additional fitting parameters could, for example, be the weighing factors for the individual models in an effective grain-interaction model. This fitting is possible as the grain-interaction parameters have a distinct influence on the diffraction stress factors. Non-linear $\sin ^{2} \psi$-plots are characteristic for the occurrence of anisotropic grain interaction. It is recommended to use measured data from various reflections simultaneously in the fitting, which can considerably support the uniqueness of the obtained fit results.

\section{Results and discussion}

\section{Mechanical elastic constants}

Components of the mechanical stiffness tensor calculated employing various graininteraction models have been gathered in table 1. The stiffness tensor corresponds to transverse elastic isotropy and thus consists of five independent components (it holds that

Table 1: Independent components of the mechanical stiffness tensor of copper in Voigt notation (singlecrystal elastic stiffnesses taken from [9]; unit = GPa).

\begin{tabular}{|l|l|l|l|l|l|}
\hline $\mathbf{C u}$ & $\mathbf{C}_{11}^{S}$ & $\mathbf{C}_{33}^{S}$ & $\mathbf{C}_{44}^{S}$ & $\mathbf{C}_{12}^{S}$ & $\mathbf{C}_{13}^{S}$ \\
\hline $\mathrm{VW}$ & 202.39 & 198.88 & 47.16 & 102.65 & 106.16 \\
\hline $\mathrm{EK}(\eta=0.1)$ & 202.16 & 199.43 & 47.41 & 103.15 & 105.89 \\
\hline $\mathrm{EK}(\eta=1)$ & 201.30 & 201.30 & 48.17 & 104.95 & 104.95 \\
\hline $\mathrm{EK}(\eta=10)$ & 200.84 & 202.15 & 48.49 & 105.84 & 104.52 \\
\hline $\mathrm{iVW}$ & 200.80 & 204.08 & 49.56 & 106.84 & 103.56 \\
\hline
\end{tabular}

$$
\mathbf{C}_{22}^{S}=\mathbf{C}_{11}^{S}, \quad \mathbf{C}_{23}^{S}=\mathbf{C}_{13}^{S}, \quad \mathbf{C}_{55}^{S}=\mathbf{C}_{44}^{S} \quad \text { and }
$$
$\mathbf{C}_{66}^{S}=1 / 2\left(\mathbf{C}_{11}^{S}-\mathbf{C}_{12}^{S}\right)$ ) for cases of anisotropic grain interaction. For the traditional Eshelby-Kröner model $\quad(\eta=1), \quad$ an isotropic mechanical stiffness tensor is obtained, i.e. only two independent components occur $\left(\mathbf{C}_{11}^{S}, \mathbf{C}_{12}^{s}\right)$. It can be concluded that the effect of the grain interaction on the mechanical elastic constants is relatively weak. 


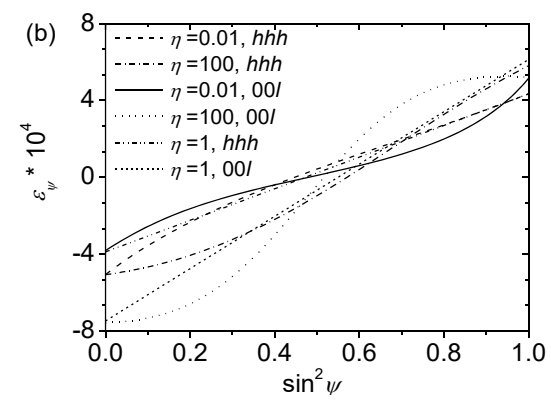

Figure 1: $\sin ^{2} \psi$-plots for the hhh and $00 \mathrm{l}$ reflections of a crystallographically untextured copper polycrystal subjected to a plane-rotationally symmetric state of stress (100MPa) according to the (a) Vook-Witt $(V W)$ and inverse Vook-Witt (iVW) models and (b) Eshelby-Kröner model involving different ellipsoidal crystallite morphologies (single-crystal elastic stiffnesses taken from [9]).

\section{Diffraction stress factors}

The effect of the type of grain interaction on the diffraction (X-ray) stress factors will be demonstrated using plots of lattice strain $\varepsilon(h k l, \varphi, \psi)$ versus $\sin ^{2} \psi$ (so-called $\sin ^{2} \psi$ plots) assuming that the polycrystal considered is subjected to a planar, rotationally symmetric state of stress $\left(\left\langle\sigma_{11}^{S}\right\rangle=\left\langle\sigma_{22}\right\rangle=\sigma_{\|}\right)$.

$\operatorname{Sin}^{2} \psi$-plots

calculated employing the different graininteraction models are shown in figure 1.

The macroscopic elastic anisotropy (transverse isotropy) is reflected by the occurrence of non-linear $\sin ^{2} \psi$-plots. Only for the traditional Eshelby-Kröner model ( $\eta=1$; spherical grain morphology), linear $\sin ^{2} \psi$-plots occur, as in this case, the polycrystal presents macroscopic isotropy. It can be concluded from figure 1 that the effect of the grain interaction on the diffraction stress factors generally is much more pronounced than the effect on mechanical elastic constants.

\section{Comparison of the direction-dependent grain-interaction models [11]}

Similarities in the results obtained for both the mechanical elastic constants and the diffraction stress factors from the different grain-interaction models occur. It has been found both on the basis of numerical calculations, analytical simplifications of certain tensors involved in the Eshelby-Kröner model and plausibility considerations, that the grain-

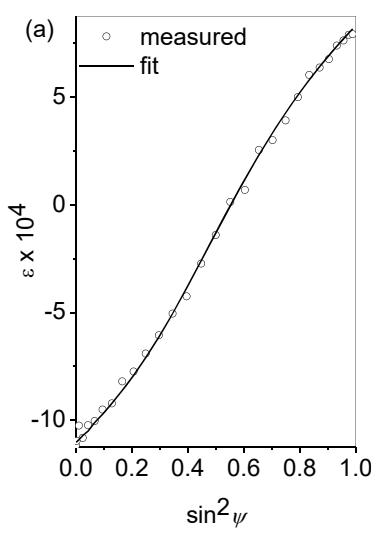

Figure 3: $\sin ^{2} \psi$-plots for the 002 reflections of a copper thin film fitted employing grain-interaction models accounting for surface anisotropy(a) and grain-shape texture (b) (for details, see [12]).

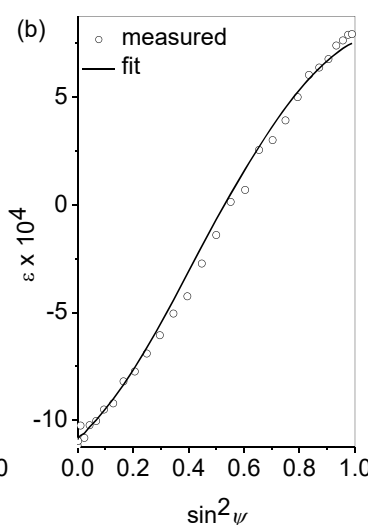

interaction assumptions in the Eshelby-Kröner model are similar to those of the Vook-Witt model if disc-like grain morphology is considered, whereas the graininteraction assumptions in the Eshelby-Kröner model are similar to those of the inverse Vook-Witt model if needle-like grain morphology is considered. These findings imply that for certain (hypothetical) cases, the effect of surface anisotropy on elastic properties of polycrystals cannot be distinguished from the effect of a grain-shape texture.

\section{An experimental example}

Six reflections of a sputter-deposited copper layer of 
thickness 500nm have been fitted simultaneously employing the above explained strategy for stress analysis (see figure 3, showing exemplary $\sin ^{2} \psi$-plots for the 200 reflections; [12]). A better fit to the experimental data has been obtained for the case of surface anisotropy. These findings are in line with the results of microstructural investigations. The specimen has a columnar microstructure with an average grain-aspect ratio close to one. Thus, surface anisotropy occurs.

\section{Summary}

(i) The traditional grain-interaction models due to Voigt, Reuss, Neerfeld-Hill and EshelbyKröner are inappropriate for the stress analysis of specimens exhibiting direction-dependent grain interaction.

(ii) Thin films are generally mechanically elastically anisotropic also in the absence of crystallographic texture, due to the occurrence of surface anisotropy. The elastic grain interaction of such polycrystals can be modelled employing an effective grain-interaction model combining the extreme Reuss, Voigt, Vook-Witt and inverse Vook-Witt models.

(iii) Polycrystals with a grain-shape (morphological) texture are mechanically elastically anisotropic also in the absence of crystallographic texture. Their elastic grain interaction can be modelled employing an extension of the traditional Eshelby-Kröner model.

(iv) For extreme grain-shape textures (discs and needles, parallel and perpendicular to the surface, respectively), the grain interaction in the Eshelby-Kröner model exhibits similarities with the grain interaction in the Vook-Witt and inverse Vook-Witt models, respectively.

(v) (X-ray) Diffraction is a sensitive tool to investigate elastic grain interaction. Graininteraction parameters can be refined in a stress analysis together with the unknown stress tensor components.

\section{References}

1. Hauk, V. (editor), 1997, Structural and residual stress analysis by nondestructive methods (Amsterdam: Elsevier).

2. Welzel, U. \& Mittemeijer, E. J., 2003, J. Appl. Phys., 93, 9001.

3. van Leeuwen, M., Kamminga, J.-D. \& Mittemeijer, E. J., 1999, J. Appl. Phys., 86, 1904.

4. Leoni, M., Welzel, U., Lamparter, P., Mittemeijer, E.J.\& Kamminga, J.-D., 2001, Phil. Mag. A, 81, 597.

5. Welzel, U., Leoni, M. \& Mittemeijer, E. J., 2003, Phil. Mag. 83, 603.

6. Welzel, U., Leoni, M. \& Mittemeijer, E.J., 2004, in: Diffraction analysis of the microstructure of materials, edited by Mittemeijer, E.J. \& Scardi, P. (Springer, Berlin), pp. 363-390.

7. Welzel, U. \& Fréour, S. in preparation.

8. Vook, R.W. \& Witt, F., 1965, J. Appl. Phys., 36, 2169.

9. Koch, N., Welzel, U., Wern, H. \& Mittemeijer, E. J., 2004, accepted for publication in Phil. Mag. 
10. Meyers, M. A. \& Chawla, K. K., 1984, Mechanical metallurgy, principles and applications (Englewood Cliffs, New Jersey: Prentice-Hall), p. 57.

11. Welzel, U., Fréour, S. \& Mittemeijer, E.J., in preparation.

12. Kumar, A., Welzel, U., Fréour, S. \& Mittemeijer, E.J., in preparation. 